Results: Eighty-five patients were first diagnosed with SLE wherein their average age was 28.10 years old $\pm 12.03,34.12 \%$ had hypertension, and $74.12 \%$ with renal involvement. The patients' cumulative 10 -year survival was $75 \%$ with average survival time of 9.84 years. Moreover, biopsy- proven lupus nephritis had significantly longer survival time (mean $=10.57$ years, $p=0.006$ ) while those with cardiopulmonary manifestations had shorter survival (mean $=0.71$ years, $\mathrm{p}=0.030$ ) as well as those on hemodialysis (mean $=8.82$ years, $\mathrm{p}=0.040$ ). Lastly, eleven patients $(12.94 \%)$ expired during the study period with active diseases and infections as the common causes of mortality.

Figure 1. Kaplan-Meier Curve showing the survival of patients with SLE from 2004 to 2014 (Mean=9.842, Median=8.625)
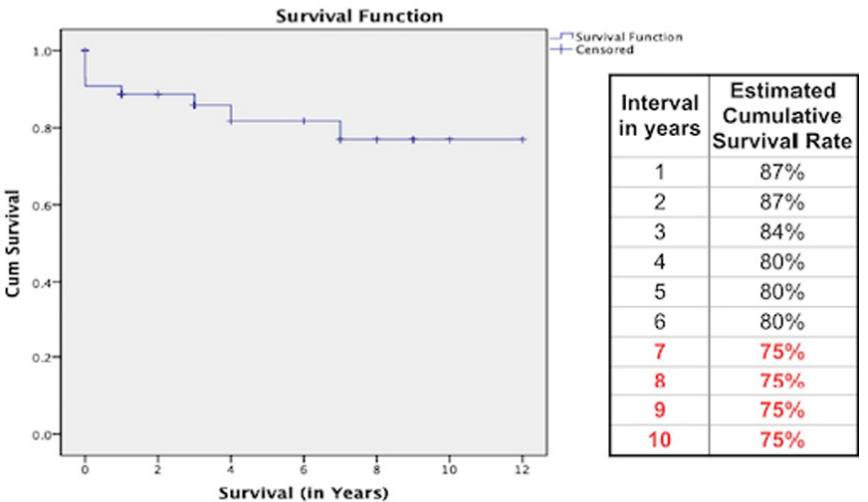

Conclusions: The 10-year survival rate of patients with systemic lupus erythematosus was $75 \%$ which was comparable to the findings from several countries. Although renal involvement was the most common initial manifestation, it did not significantly affect survival similar to prominent studies.

Disclosure of Interest: None declared

DOI: 10.1136/annrheumdis-2017-eular.1303

\section{SAT0281 JOINT ACTIVITY INDICES CORRELATES WITH ULTRASONOGRAPHIC SCORE IN SLE PATIENTS WITH MUSCULO-SKELETAL INVOLVEMENT}

E. Cipriano, F. Ceccarelli, C. Perricone, L. Massaro, G. Capalbo, F. Natalucci, F.R. Spinelli, F. Miranda, S. Truglia, C. Alessandri, G. Valesini, F. Conti. Medicina Interna e Specialità Mediche, Sapienza Università di Roma, Roma, Italy

Background: Joint involvement represents one of the most frequent manifestations in Systemic Lupus Erythematosus (SLE) patients (incidence 69-95\%), with different degrees of severity. Currently, there are no validated indices to evaluate joint involvement in SLE. Musculo-skeletal ultrasonography (US) has been widely applied in patients affected by different arthropathies. US-detected synovitis reflects the inflammatory state at the joint level, as demonstrated by the correlation with histological modifications. Furthermore, US-synovitis significantly correlated with disease activity indices, such as DAS28.

Objectives: In the present study, we aimed at assessing a correlation between the composite indices DAS28 (Disease Activity Score 28), CDAI (Clinical Disease Activity Index), SDAI (Simplified Disease Activity Index), STR (Swollen to Tender Ratio) and the US-detected synovitis in a cohort of SLE patients with joint involvement.

Methods: One hundred seven patients (M/F 7/100, mean age \pm SD $48.4 \pm 13.8$ years, mean disease duration \pm SD $156.0 \pm 129.6$ months) with at least one tender joint were enrolled. We registered the number of swollen and tender joint count (0-28) and the patient's/physician's disease activity on visual analogue scale (0-100). DAS28-ESR, CDAI, SDAI and STR were calculated. The US evaluation of 12 joints (I-V metacarpophalangeal, I-V proximal interphalangeal, wrist and knee bilateral) was performed to identify inflammatory features (synovial effusion, synovial hypertrophy and power Doppler) according with OMERACT definitions. These elementary lesions were scored according to a semi-quantitative scale $(0=$ absent, $1=$ mild, $2=$ moderate and $3=$ severe $)$. The sum of the semiquantitative scores allows obtaining a total score of the patient's inflammatory state $(0-216)$.

Results: As reported in Figure 1, by using the Spearman analysis, a positive correlation between US-score and SDAI $(r=0.33, P=0.02)$, CDAI $(r=0.29, P=0.03)$ and STR $(r=0.42, P=0.0005)$ was identified. In particular, SLE patients with high disease activity according with STR value $(>1)$ showed a higher US score (16.3 \pm 19.3$)$ in comparison with moderate $(7.7 \pm 4.5, \mathrm{P}=\mathrm{NS})$ or low disease activity (7.1 $\pm 7.9, P=0.02)$. Moreover, US score resulted significantly lower in patients with DAS28 remission compared to those with an active disease $(4.5 \pm 4.4$ versus 7.05 $\pm 5.1, P=0.03$; Mann-Whitney test).

Conclusions: We analyzed a large SLE cohort with articular involvement identifying a significant correlation between US scoreand the composite indices CDAI, SDAI and STR. Furthermore, US-score may be able to discriminate DAS28-remission patients. Taken together, these results suggest the ability of composite indices in detecting the joint activity in SLE patients and the possibility
Figure 1: correlation between US score and SDA, CDAI, STR
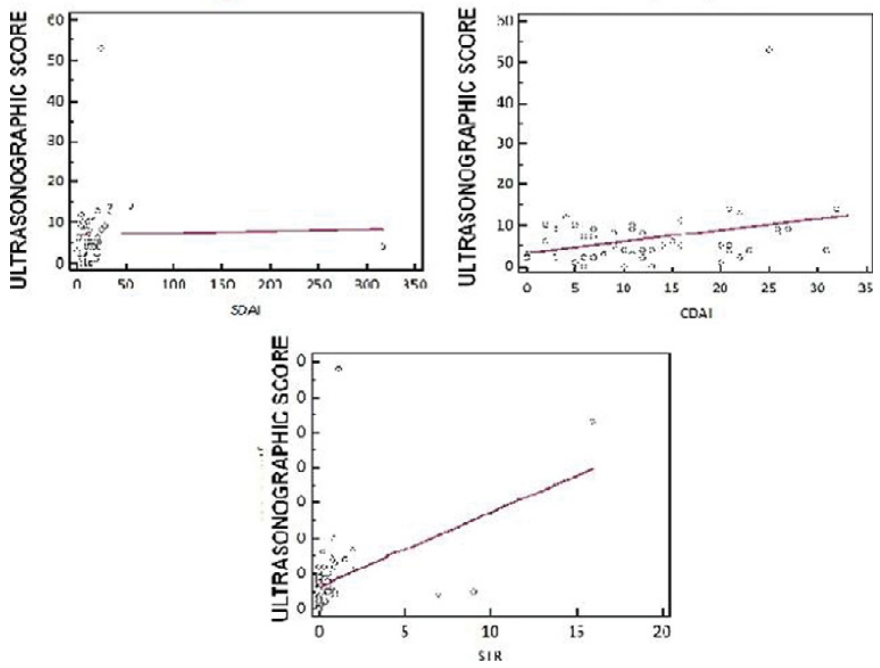

to use them in clinical practice to assess this frequent and potentially disabling manifestation

Disclosure of Interest: None declared

DOI: 10.1136/annrheumdis-2017-eular.5231

\section{SAT0282 AUTOIMMUNITY AND PREGNANCY: EVIDENCE FROM AN OBSERVATIONAL STUDY}

F. Del Porto ${ }^{1}$, S. Ferrero ${ }^{2}$, M. Proietta ${ }^{3}$, N. Cifani ${ }^{3}$, M. Catoni ${ }^{1}$, F.M. Ubaldi ${ }^{2}$, L. Rienzi ${ }^{2}$, L. Ferri ${ }^{1}$, R. Di Rosa ${ }^{3} .{ }^{1}$ SApienza University; ${ }^{2}$ Genera Valle Giulia, Roma; ${ }^{3}$ SApienza University, Rome, Italy

Background: Obstetrical APS is defined by positive aPLs and a history of one or more unexplained deaths of morphologically normal fetus at or beyond the 10th week of gestation (WG) or one or more consecutive spontaneous abortions before the 10th WG. Also one or more premature birth before 34 WG because of eclampsia, severe pre-eclampsia, or recognized features of placental insufficiency represent one of the diagnostic criteria (1). Infertility is defined as the inability of a couple practicing frequent intercourse and not using contraception to conceive a child after 12 months. Autoimmune diseases are not inclued among major causes of infertility, despite defective embryonic implantation could be considered an aspect of recurrent fetal losses in patients with positive antiphospholipid antibodies, due to their capabilities to reduce trophoblast prolipheration and growth (2).

Objectives: The aim of our study was to evaluate the prevalence of aPLs and pregnancy outcome in a population of women undergoing in vitro fertilization. Methods: from December 2012 to December 2016, we selected 75 consecutive patients undergoing in vitro fertilization and evidence positive autoantibodies. Each of them was evaluated for genetic, anatomic, hormonal and infective causes of infertility. Moreover antinuclear antibodies (ANA), anticardiolipin antibodies (aCL), anti-p2-glycoprotein I (GP1), lupus anticoagulant (LA) and extractable nuclear antigens (ENA) profile were assessed.

Results: patients mean age was $41.38 \pm 4.87$ years, ranging from 31 to 53 years. Prevalence of aPLs in our population was $68 \%$. All women showed at least twice positive aPLs. aCL IgM and LA were the main antibody populations observed. ANA were positive in $50 \%$ of women, whereas SSA or SSB were positive in $4.17 \%$. In $22.9 \%$ of patients a systemic autoimmune disease was newly diagnosed, mainly systemic lupus erythematosus. All patients with hystory of recurrent miscarriages and positive $\mathrm{aPL}$ were treated with subcutaneous low weight heparin plus daily oral acetylsalicylic acid (ASA $100 \mathrm{mg}$ ) (3). Full term pregnancy was obtained in $45.8 \%$ of patients.

Conclusions: Prevalence of aPLs in the general population is $1-5 \%$, whereas in our selected series positive aPLs were detected in $68 \%$ of the patients, suggesting that prevalence of aPLs may be increased in infertile patients (4). Moreover, a systemic autoimmune disease was newly diagnosed in $22.9 \%$ of patients suggesting that paucisimptomatic disease can be underestimated. Finally we would suggest that treating autoimmune co-morbidities ameliorates implantation rates in women undergoing in vitro fertilization (5)

References:

[1] Miyakis S, Lockshin MD, Atsumi T, et al. J Thromb Haemost 2006; 4:295-306.

[2] Chinhizola CB, de Jesus GR.A. Lupus 2014; 23:1232-1238.

[3] Proietta M, Ferrero S, Del Porto F. Lupus 2014;23:724-725.

[4] AndreoliL, Bertsias GK, Agmon-Levin N et al. Ann Rheum Dis. 2016;30:133148

[5] Kocku A, Yavuz E, CeliK H,Bildircin D. Arch Gynecol Obstet 2012;286:12831289.

Disclosure of Interest: None declared

DOI: 10.1136/annrheumdis-2017-eular.6425 